\title{
Combination of Pseudoephedrine and Emodin Ameliorates LPS-Induced Acute Lung Injury by Regulating Macrophage M1/M2 Polarization Through the VIP/CAMP/PKA Pathway
}

\section{Shu-Guang Yan}

Shaanxi University of Chinese Medicine

WenBa Wang

Shaanxi University of Chinese Medicine https://orcid.org/0000-0002-7319-4200

Jing-Tao Li

Shaanxi University of Chinese Medicine

Yi Hui

Shaanxi University of Chinese Medicine

Jie Shi

Shaanxi University of Chinese Medicine

Xu-Yan Wang (D 4067010@qq.com)

Genetics and Metabolism, Xi 'an Children's Hospital

\section{Research}

Keywords: acute lung injury, LPS, Pseudoephedrine+emodin, macrophages, VIP

Posted Date: July 13th, 2021

DOI: https://doi.org/10.21203/rs.3.rs-680435/v1

License: (c) (i) This work is licensed under a Creative Commons Attribution 4.0 International License.

Read Full License 


\section{Abstract}

Background: Acute lung injury (ALI) is an acute multifactorial infectious disease induced by trauma, pneumonia, shock, and sepsis. This study aimed to investigate the protective effects of pseudoephedrine and emodin combined treatment in experimental ALI, as well as the mechanisms underlying the regulation of inflammation and pulmonary edema via the VIP /CAMP/PKA pathway.

Methods: Wistar rats were divided into a control group, lipopolysaccharide(LPS) group, and a pseudoephedrine + emodin treatment group. Rats in the control group were given intraperitoneal saline injections, while rats in the LPS and treatment groups were given intraperitoneal LPS injections to induce ALI. After being treated with pseudoephedrine and emodin for 24 hours, all animals were sacrifce. Anal temperatures were taken on an hourly basis for eight hours after LPS injection. Pathological examination of lung specimen was performed by H\&E staining. Cytokines (IL-1 $\beta$, TNF- $a$, IL-6, IL-10, Arg-1, CD86, CD80, CD206, F4/80, VIP) in colon were assayed by ELISA and immunofuorescence. The expression of VIP, CAMP, AQP-1, AQP-5, p-PKA/PKA, p-IKBa/IKBa, and p-p65/p65 protein in lung was determined by western blotting.

Results: After rats being treated with pseudoephedrine + emodin, the symptoms (febrility) of ALI were alleviated. The contents of infammatory cytokines (IL-1 $\beta$, TNF- $a$, IL-6) were decreased and antiinflammatory cytokines(IL-10, Arg-1) were signifcantly increased in serum. Pseudoephedrine + emodin treatment effectively promoted VIP CAMP and p-PKA protein expression in lung tissues, and significantly inhibited NF-KB phosphorylation,Pseudoephedrine + emodin treatment can inhibit M1 polarization and promoted M2 polarization via the VIP/cAMP/PKA signaling pathway.

Conclusions: The combination of pseudoephedrine hydrochloride and emodin was effective in ameliorating LPS-induced ALI in rats by inducing VIP/CAMP/PKA signaling, inhibiting the NF-KB inflammatory pathway, suppressing macrophage M1 polarization, and promoting macrophage $M 2$ polarization.

\section{Background}

ALI is an acute multifactorial infectious disease induced by trauma, pneumonia, shock, and sepsis (Ware et al., 2000; Gupta et al., 2007). The mortality rate of ALI is $30 \%-50 \%$ (Derwall et al., 2018), and survivors live with significantly compromised quality of life resulting from severe damage to lung functions (Matthay et al., 2019). ALI is characterized by inflammatory cell infiltration, massive production of inflammatory mediators, and diffuse lung inflammation (Shi et al., 2014). It is recognized as one of the most direct causes of death in patients with sepsis, in which the intestinal barrier function is impaired and a large number of bacteria and endotoxins in the intestine invade the circulation through the portal vein and lymphatic system, thereby causing lung and intestinal infections and triggering multi-organ dysfunction syndrome (Karpurapu et al., 2018; Liu et al., 2018). 
ALI rats are commonly used as animal models in pharmacological studies of lipopolysaccharide (LPS)induced ALI. It is widely known that LPS activates the expression of inflammatory cytokines, such as interleukin-6 (IL-6) and tumor necrosis factor-a (TNF-a), and induces NF-kB signaling, ultimately leading to cytokine storm and endotoxic shock (Huang et al., 2017). The histological nature of ALIs was initially considered to be diffuse alveolar damage; this has now been supplemented with capillary congestion, pulmonary atelectasis, intra-alveolar hemorrhage, alveolar edema, as well as the resulting proliferation of epithelial cells and interstitial pulmonary edema, with particular emphasis on alveolar and interstitial edema triggered by inflammatory exudation as an important pathological aspect of ALI (Taylor et al., 2017). Macrophages are central nodes of immune activity that demonstrate high plasticity and versatility in response to changes in the body's internal environment; they can exhibit both pro-inflammatory and anti-inflammatory properties in response to LPS stimulation (Locati et al., 2019). Depending on the stage of ALI development, macrophages could be polarized to one of two different phenotypes: classically activated macrophage M1, or alternatively activated macrophage M2. During the early stages of inflammation, M1-mediated NF-KB signaling induces the release of several cytokines, including TNF-a, IL6, IL-1 $\beta$, chemokines, and granulocyte colony-stimulating factors (G-CSF) (Murray., 2016). M1 macrophages can further differentiate into $\mathrm{TH} 1$ and $\mathrm{TH} 17$ pro-inflammatory cells, which increases inflammatory response, but excessive production of pro-inflammatory factors, reactive oxygen species, and reactive nitrogen species could lead to tissue damage (Wiersinga et al., 2019). During the inflammation suppression stage, macrophages are typically polarized into M2 macrophages, which have anti-inflammatory repair functions and are typically induced by classical TH2 cytokines, such as IL-4, IL10 and glucocorticoids.

Vasoactive intestinal peptide (VIP) is one of the most important immunoreactive neuropeptides in the lung; it has several notable biological effects, such as reducing granulocyte recruitment (Onoue et al., 2013) and promoting the expression of the anti-inflammatory factors IL-4 and IL-10 (Xu et al., 2014). VIP agonists have been found to exhibit protective properties when used to treat asthma, pulmonary hypertension, chronic bronchitis and pulmonary fibrosis (Laube., 2015). Cyclic adenosine monophosphate (CAMP) is an important substance for tissue and cell metabolism and the maintenance of physiological functions; it can activated through different means, of which hormones and neurotransmitters are some of the most important. As an immunoneuropeptide, VIP can bind to G proteincoupled receptors to activate adenylate cyclases (ACs) and promote cAMP release (Pearce et al., 2010). Protein kinase (PKA) is a major CAMP target and cAMP can bind to the regulatory subunit of PKA to induce PKA phosphorylation, thereby forming the VIP/CAMP/PKA signaling pathway (Juana et al., 2013).

In traditional Chinese Medicine, connections between the physiological functions of the lung and the intestines are often emphasized when treating diseases of the lung and intestine. This treatment approach, which translates to "lung-intestine combinational therapy", tends to employ herbal preparations made from rhubarb and Ephedra sinica. The dried stems and leaves of ephedra are traditionally prescribed as a treatment for cough and asthma (Soni et al., 2004); the herb exhibits a certain degree of toxicity, but it remains popular in China and Japan as a remedy for symptoms related to upper respiratory tract infections - especially cough and fever (Ma et al., 2014; Lin et al., 2016). One of 
main active ingredients of ephedra is pseudoephedrine, which produces notable anti-inflammatory effects (Li et al., 2019). Studies have also demonstrated that pseudoephedrine, when taken after antibiotics as an adjuvant treatment for symptom relief by patients with acute respiratory infections (ARIs), was effective in improving respiratory symptoms, especially those of nasal congestion and sinus headache (LaForce et al, 2008). The main active component of rhubarbs is emodin, which is a natural anthraquinone derivative. Recent studies have revealed a wide range of pharmacological properties of emodin, including anticancer, hepatoprotective, anti-inflammatory, antioxidant, and antibacterial properties (Dong et al, 2016). We conducted preliminary experiments in which ALI rats were treated with ephedra and rhubarb through gavage administration and discovered significant suppression of inflammation and pulmonary edema. We hypothesized that this could be related to elevated VIP expression in the serum and bronchoalveolar lavage fluid (BALF) and endeavored to further investigate the protective effects of this pseudoephedrine + emodin combination on rats with LPS-induced ALI, as well as the mechanisms underlying the regulation of inflammation and pulmonary edema via the VIP /CAMP/PKA pathway.

\section{METHODS}

\section{Chemicals and reagents}

Pseudoephedrine and emodin (purity $>99 \%$ ) were purchased from Baoji Chenguang biology Ltd. LPS was purchased from Sigma-Aldrich Co. ELISA kits for IL-1 $\beta$, TNF-a, IL-10, Arg-1 and VIP were obtained from Elabscience Biotechnology Co. Ltd. (Wuhan, China). Antibodies against AQP-1, AQP-5 VIP, CAMP, PKA/phospho-PKA,IKBa/phospho-IKBa, NF-KB p65/phospho-NF-KB p65, and $\beta$-actin were purchased from Affinity Biosciences Ltd. (OH, USA). HRP-conjugated secondary antibodies were purchased from Wuhan Boster Biological Technology LTD. (Wuhan, China). Trizol and Western blot chemiluminescence reagents were obtained from Ambion Thermo Fisher Scientific - CN (Shanghai, China).

\section{Animal model construction and sampling}

Wistar rats were divided into a control group $(n=5)$ LPS group $(n=5)$, and a pseudoephedrine + emodin treatment group $(n=5)$. Rats in the control group were given intraperitoneal saline injections, while rats in the LPS and treatment groups were given intraperitoneal LPS injections $(10 \mathrm{mg} / \mathrm{kg})$ to induce ALI. Gavage was then performed in each group at 0,8 and 16 hours after LPS injection: those in the control and LPS groups were given physiological saline while those in the treatment group were given pseudoephedrine $(30 \mathrm{mg} / \mathrm{kg})$ and emodin $(30 \mathrm{mg} / \mathrm{kg})$. Anal temperatures were taken on an hourly basis for eight hours after LPS injection. Rats were sacrificed 24 hours after LPS injection through intraperitoneal injections of $10 \%$ chloral hydrate $(3 \mathrm{~mL} / \mathrm{kg})$. Left ventricular arterial blood was extracted from the sacrificed rats and centrifuged at $3000 \mathrm{~g}$ for 10 minutes; the extracted serum was stored at -80 ${ }^{\circ} \mathrm{C}$. D/W ratios in the lower lobe of the right lung were assessed, and the middle lobes of the right lung were fixed with $4 \%$ paraformaldehyde for pathological staining, immunohistochemistry, and immunofluorescence analysis. The remaining lung tissues were stored at $-80^{\circ} \mathrm{C}$ for further analysis. 


\section{Isolation and culture of alveolar macrophages from rat BALF}

A male Wistar rat weighing $200-250 \mathrm{~g}$ was anesthetized with $1.5 \%$ sodium pentobarbital $(35 \mathrm{mg} / \mathrm{kg})$ and fixed on a rat plate. The neck was disinfected in accordance with routine procedures, and the skin of the neck was incised to fully expose the trachea; an incision was made in the middle of the trachea, followed by surgical intubation and fixing. The lung was washed ten times; during each lavage, $6 \mathrm{ml}$ of saline was injected into the lung cavity, the lung was gently rubbed, then lavage fluid was withdrawn. BALF was collected and filtered through a 200 mesh filter. The filtrate was centrifuged at $1000 \mathrm{~g}$ for $10 \mathrm{~min}$ and the supernatant was discarded. The pellet was washed twice by centrifugation with PBS at $1000 \mathrm{~g}$ for 10 min. These cells were seeded in sterile 6-well culture dishes and incubated at $37^{\circ} \mathrm{C}$ in an incubator containing 5\% $\mathrm{CO} 2$.

\section{Histopathological analysis}

Lung tissues were fixed with $4 \%$ paraformaldehyde; these were embedded in paraffin sections $(2 \mu \mathrm{m}$ thick), de-paraffinized, and then rehydrated with an ethanol gradient. Lung tissues were treated with hematoxylin and eosin (H\&E) for staining and photographed under light microscopy. The results were graded on a scale of 0 to 3 points by an experienced pathologist in accordance with established procedure from the literature (Szapiel et al., 1979).

\section{Changes in anal temperature and thermal response index (TRI)}

Rat body temperatures were measured on an hourly basis for eight hours after LPS injection; these were calculated as $\Delta \mathrm{T}$. Thermal response index $\left(\mathrm{TRI},{ }^{\circ} \mathrm{C} \times \mathrm{h}\right)$ was calculated based on the area between the temperature-time curve and the x-axis (Lu et al., 2018); specifically, it was calculated as the summation of the area of each trapezoid under every two data points. The area of each such trapezoid from $t_{i}$ to $t_{i+1}$ was calculated as $\left(\mathrm{t}_{\mathrm{i}+1}-\mathrm{t}_{\mathrm{i}}\right) \times\left(\Delta \mathrm{T}_{\mathrm{i}}+\Delta \mathrm{T}_{\mathrm{i}+1}\right) / 2$.

\section{Lung dry/wet (D/W) weight ratio}

Samples from the lower lobe of the right lung were collected 24 hours after LPS stimulation, blotted with filter paper to remove excess water and weighed immediately (wet weight), and then dried continuously in an oven at $80^{\circ} \mathrm{C}$ for 48 hours to obtain the dry weight. Tissue edema was assessed by the ratio of wet lung weight to dry lung weight.

\section{Enzyme-linked immunosorbent assay for the quantification of cytokines}

Levels of IL-1 $\beta$, TNF-a, IL-6, IL-10, Arg-1 and VIP in rat serum and cultured macrophages were measured by ELISA kits. The optical density of the microtiter plate was read at $450 \mathrm{~nm}$.

Immunohistochemical analysis 
Paraffin sections ( $3 \mu \mathrm{m}$ thick) were de-paraffinized and then rehydrated with an ethanol gradient. Sections were incubated with $3 \% \mathrm{H}_{2} \mathrm{O}_{2}$ to remove endogenous peroxidase, followed by incubation with anti-F4/80 antibodies $\left(1: 100\right.$, rabbit) overnight at $4^{\circ} \mathrm{C}$. It was then further incubated with HRP-conjugated secondary antibodies for $30 \mathrm{~min}$ at $37^{\circ} \mathrm{C}$, stained with hematoxylin, and photographed under sealed conditions.

\section{Immunofluorescence analysis}

Cell slides were de-paraffinized, rehydrated with an ethanol gradient, and 50\% TritonX was added dropwise to the tissue and left for 10 minutes at room temperature. They were then washed with PBS thrice with five minutes each time. Citrate buffer was added to the slide and antigen retrieval was carried out at high temperature for 5 minutes. The slides were washed with $0.01 \mathrm{M}$ PBS and closed with goat serum for $10 \mathrm{~min}$ at room temperature. Sections were incubated overnight at $4^{\circ} \mathrm{C}$ with primary antibodies anti-CD86 (1:50; BIOSS, BeiJing, China), anti-IL-6 (1:50; BIOSS, BeiJing, China), anti-CD206 (1:50; Abcam, ShangHai, China), anti- IL-10 (1:50; Abcam, ShangHai, China). Unbound primary antibodies were washed off with PBS, and sections were incubated with secondary antibodies followed by nuclear staining and image acquisition. Lung tissue sections were incubated with primary antibodies anti-CD80 (1:50; BOSTER, Wuhan, Hubei,China), anti-IL-1 $\beta$ (1:100; BOSTER, Wuhan, Hubei,China), anti-IL-10 (1:50; BOSTER, Wuhan, Hubei,China) and anti-F4/80 (1:100; BOSTER, Wuhan, Hubei,China). Subsequent steps were the same for cellular immunofluorescence.

\section{Western blot analysis}

Lung tissue samples were lysed in RIPA buffer containing protease and phosphatase inhibitors for 30 min. Protein concentrations were measured using the BCA assay kit (Beyotime, China). Proteins were denatured by heating in a $95^{\circ} \mathrm{C}$ water bath. Electrophoresis was carried out to transfer proteins to PVDF membranes. Membranes were incubated overnight at $4^{\circ} \mathrm{C}$ with the primary antibodies $\beta$-actin, VIP, CAMP, AQP-1, AQP-5, PKA, p-PKA, IKBa, p-IKBa, p65, and p-p65, then washed thoroughly three times with PBST and incubated with HRP-conjugated secondary antibodies (1:5000 dilution) for 1 hour at room temperature. Bands were detected with ECL (AmershamPharmacia Biotech, Piscataway, NJ) and the intensity of the bands was quantified using the Image-J gel analysis software.

\section{Real-time PCR analysis}

Total RNA was extracted from cells using Trizol reagent (Ambion, China). cDNA was reverse transcribed using TransScript First-strand cDNA Synthesis Kit (VAZYME, China) and stored at $-80^{\circ} \mathrm{C}$. Relative gene expression was quantified with QuantStudio 6 ( $\mathrm{ABI}, \mathrm{USA})$ and $0.4 \mu \mathrm{g}$ of total RNA was reverse transcribed under the following PCR reaction system: $\triangle$ Pre-denaturation: $95^{\circ} \mathrm{C}, 10 \mathrm{~min}, 1 \mathrm{cycle}$. $\otimes$ Denaturation: $95^{\circ} \mathrm{C}$, $15 \mathrm{~s}, 40$ cycles. $\triangle A$ Annealing and extension: $60^{\circ} \mathrm{C}, 60 \mathrm{~s}, 40$ cycles. The following primers were used: GAPDH, forward 5'-ATGGGTGTGAACCACGAGA-3' and reverse 5'-CAGGGATGATGTTCTGGGCA-3', product size 229 BP; IL-6, forward 5'-CACAGAGGATACCACTCCCAACAGA-3' and reverse 5'-

ACAATCAGAATTGCCATTGCACAAC-3', product size 124 BP; TNF-a, forward 5'AGCACAGAAAGCATGATCCG-3' and reverse 5'-CTGATGAGAGGGAGGCCATT-3', product size 212 BP; iNOS, 
forward 5'-TTGGCTCCAGCATGTACCCT-3' and reverse 5'-TCCTGCCCACTGAGTTCGTC-3', product size 121 BP; IL-10, forward 5'-GCTGGACAACATACTGCTAACCG-3' and reverse 5'-CACAGGGGAGAAATCGATGACAG3', product size 218 BP; Arginase-1, forward 5'-ATCGTGTACATTGGCTTGCG-3' and reverse 5'CGTCGACATCAAAGCTCAGG-3', product size 184 BP; Ym-1, forward 5'TGGAATTGGTGCCCCTACAA-3' and reverse 5'-CCACGGCACCTCCTAAATTG-3', product size 239 BP. Lung tissue RNA were extracted using the same steps as the those for the cells, but using the following primers: $\beta$-actin, forward $5^{\prime}$ CACGATGGAGGGGCCGGACTCATC-3' and reverse 5'-TAAAGACCTCTATGCCAACACAGT-3', product size 240 BP, TNF-a, forward 5'CCGATTTGCCATTTCATACCAG-3' and reverse 5'-

TCACAGAGCAATGACTCCAAAG-3', product size 232 BP, IL-1 $\beta$, forward 5'-CAGGTCGTCATCATCCC-3' and reverse 5'-TCAAATCTCACAGCAGCAT-3', product size 190 BP, NLRP3, forward 5'-

CTGCTGAAGTGGATCGAAGTG-3' and reverse 5'-TGCAAAAGGAAGAAACCACGT-3', product size 187 BP, VIP, forward 5'-ATCCAGAAGCAAGCCTCAGT-3' and reverse 5'-ATAGGGCGTGTCATTCTCCG-3', product size 211 BP, IL-10, forward 5'-CAGTCAGCCAGACCCACAT-3' and reverse 5'-GGCAACCCAAGTAACCCT-3', product size $141 \mathrm{BP}$; Arg-1, forward 5'-GGTAGCAGAGACCCAGAAGA-3' and reverse 5'-

CAGCGGAGTGTTGATGTCAG-3', product size 151 BP. The relative amount of mRNA was calculated using the comparative $\mathrm{Ct}(\triangle \mathrm{Ct})$ method and compared with GAPDH and $\beta$-actin.

\section{Statistical analysis}

All quantitative data were expressed as mean \pm SD. Statistical analysis was carried out using SPSS 19.0 (IBM). Comparisons between experimental groups were conducted using one-way ANOVA, whereas multiple comparisons were carried out using the LSD method. Statistical significance was defined as $p<$ 0.05 or $\mathrm{p}<0.01$.

\section{Results}

\section{Pseudoephedrine + emodin inhibits secretion of pro- inflammatory factors and reduces pulmonary edema}

The chemical structures of pseudoephedrine and emodin are shown in Fig. 1A.

In the LPS group, microscopic observation of lung tissues after H\&E staining revealed thickened lung interstitium with significant neutrophil infiltration and airway hemorrhage. In comparison, lung injury was significantly ameliorated in the treatment group (Fig. 1B). Levels of inflammation were graded based on the degree of observed histological changes in lung tissues (Fig. 1C).

In the LPS group, serum ELISA results revealed significantly increased serum levels of the proinflammatory factors TNF- $\alpha$ and IL-1 $\beta$ compared with the control group (Fig. 1D-E). These levels were significantly suppressed in the treatment group. The levels of the same pro-inflammatory factors in lung tissues exhibited similar trends as those from the serum ELISA results (Fig. 1F-G). 
In the LPS group, we also found increased mRNA expression levels of inflammasome NLRP3, which is an important activator of IL-1 $\beta$. In comparison, such mRNA expression levels were suppressed in the treatment group (Fig. 1H).

In the LPS group, Western blot analysis showed suppressed expression of AQP-1 and AQP-5 proteins in lung tissues. In comparison, the expression of these proteins was significantly increased in the treatment group (Fig. 11-J).

Finally, reduced lung D/W ratio was found in the LPS group. This, too, was significantly improved after treatment with pseudoephedrine + emodin (Fig. 1K).

Collectively, these results indicate that the combination of pseudoephedrine + emodin is effective in reducing LPS-induced inflammatory injuries and pulmonary edema.

Figure 1. Effect of pseudoephedrine + emodin on histopathological changes in lung tissues (200x), expression of AQP-1 and of AQP 5, and inflammatory cytokines in LPS-induced ALI rats. (A) The chemical structures of pseudoephedrine and emodin. (B) H\&E staining. (C) Inflammation scores for histopathological changes in lung tissues after H\&E staining. (D) ELISA results for serum levels of TNF-a. (E) ELISA results for serum levels of IL-1 $\beta$. (F-H) Real-time PCR analysis results for TNF-a, IL-1 $\beta$, and NLRP3 expression levels in lung tissues. (I-J) Western blot analysis results for AQP-1 and AQP-5 protein expression levels. (K) Pulmonary edema, as reflected by $\mathrm{D} / \mathrm{W}$ weight ratios and lung weight/body weight ratios.All data are expressed as mean \pm S.D. $(n=3)$.\#\# $p<0.01$, \#\#\#p<0.001 vs. control group. * $p<$ $0.05,{ }^{* \star} p<0.01, * \star \star p<0.001$ vs. LPS group.

\section{Pseudoephedrine + emodin promotes secretion of immunosuppressive factors}

IL-10 is a common inflammatory suppressor and arginase-1 (Arg-1) is a classical marker of the alternatively activated macrophages (M2). ELISA and real-time PCR were used to measure the expression of IL-10 and Arg-1 in serum and in lung tissues (Fig. 2). In the LPS group, results revealed stress-induced increase in IL-10 and Arg-1 expression levels in both serum and lung tissues when compared to the control group. These levels were increased in the treatment group in comparison to the LPS group (Fig. 2A-D).

Figure 2. Effects of Pseudoephedrine + emodin on the expression of IL-10 and Arg-1 in LPS-induced ALI rats. (A) ELISA results for serum levels of IL-10.(B) ELISA results for serum levels of Arg-1. (C-D) IL-10 and Arg-1 lung tissue mRNA expression levels. All data are expressed as mean \pm S.D. $(n=3)$. $\# p<0.05$, \#\# $p<$ $0.01, \# \# \# p<0.001$ vs. control group. ${ }^{* *} p<0.01, * \star * p<0.001$ vs. LPS group.

\section{Pseudoephedrine + emodin affects febrility in ALI rats}

In the control group, rat body temperatures remained within normal range throughout the experiment, with no significant changes occurring within eight hours of saline injection (Fig. 3A-B). In contrast, rats in the 
LPS group exhibited significant increase in body temperature and TRI, which began as an immediate decrease within one hour after LPS injection, before significantly increasing between one to eight hours after injection. In the treatment group, the body temperatures and TRIs of the rats were significantly lower than those of the LPS group, which indicated the effectiveness of the pseudoephedrine + emodin treatment in alleviating febrility in LPS-induced ALI rats.

Figure 3. Effect of pseudoephedrine + emodin on febrility in LPS-induced ALI rats. (A-B) Changes in body temperatures and TRIs. All data are expressed as mean \pm S.D. $(n=5) . \# \# \# p<0.001$ vs. control group. ${ }^{* \star} p$ $<0.01$ vs. LPS group.

\section{VIP intervention affects pro-inflammatory and anti- inflammatory factor expression in LPS, IL-4-induced alveolar macrophages}

Macrophages play an important role in immune response. Therefore, we isolated and cultured alveolar macrophages from rats, used LPS and IL-4 to induce macrophage polarization into M1 and M2 macrophages, respectively, and then studied the expression levels of pro- and anti-inflammatory cytokines after pretreatment with different concentrations of VIP.

Figure 4 shows ELISA and real-time PCR results in measuring he expression levels of target cytokine proteins and mRNAs. In the LPS group, the expression of TNF-a, IL-6, and iNOS proteins and mRNA were significantly increased compared with the control group, while the expression of the anti-inflammatory IL10, Arg-1, and Ym-1 proteins and mRNAs were decreased; macrophage polarization towards M1 was significant. In the IL-4-induced group, the levels of pro-inflammatory-related factors were only slightly increased compared with the control group, which we hypothesize to be stress changes induced by IL-4. There were significant increases in the expression of IL-10, Arg-1, and Ym-1 proteins and mRNAs, and macrophage polarization towards M2 was significant. Increased VIP dose concentration resulted in a decrease in the expression of the pro-inflammatory TNF-a, IL-6, and iNOS proteins and mRNAs, as well as an overall increase in the expression of the anti-inflammatory IL-10, Arg-1, and Ym-1 proteins and mRNAs (Fig. 4).

These results indicate that VIP could effectively inhibit the release of pro-inflammatory factors and increase the expression of anti-inflammatory factors in macrophages.

Figure 4. Effects of VIP on levels of inflammatory and anti-inflammatory cytokines in LPS or IL-4 induced alveolar macrophages. Macrophages were pretreated with VIP $\left(10^{-6}, 10^{-7}, 10^{-8} \mathrm{~mol} / \mathrm{L}\right)$ for 24 hours, followed by LPS $(200 \mathrm{ng} / \mathrm{mL})$ or IL-4 $(40 \mathrm{ng} / \mathrm{mL})$ stimulation for 12 hours. Cells were collected and the levels of TNF-a (A), IL-6 (B), IL-10 (C), Arg-1 (D) were determined using ELISA. (E-J) TNF-a, IL-6, iNOS, IL10, Arg-1, Ym-1 mRNA expression was determined using Real-time PCR analysis. All data are expressed as mean \pm S.D. $(n=3) .{ }^{\#} p<0.05,{ }^{\# \#} p<0.01,{ }^{\# \#} p<0.001$ vs. control group. ${ }^{*} p<0.05,{ }^{\star *} p<0.01, * \star \star p<$ 0.001 vs. LPS group. $+p<0.05,++p<0.01,+++p<0.001$ vs. IL-4 group. 


\section{VIP intervention affects alveolar macrophage polarization towards $\mathrm{M} 1$ and $\mathrm{M} 2$}

It is widely known that CD80 and CD206 are surface markers of M1, M2 macrophages, respectively. We used LPS and IL-4 to induce the polarization of VIP-pretreated alveolar macrophages, followed by immunofluorescence staining for CD86, IL-6 and CD206, IL-10, respectively.

Results are shown in Fig. 5. High CD86 expression was clearly observed in alveolar macrophages after LPS induction. In contrast, M1 polarization was significantly suppressed in cells that were pretreated with VIP. Correspondingly, elevated CD206 expression was observed after IL-4 induction, and VIP pretreatment increased polarization towards M2 (Fig. 5A-G). PCR results showed similar results: compared with LPS and IL-4-groups, VIP pretreatment groups exhibited decreased CD86 mRNA expression and elevated CD206 mRNA expression (Fig. 5H-I).

These results indicate that VIP, as an immunoreactive neuropeptide, has a bidirectional regulatory effect of inhibiting M1 polarization and promoting M2 polarization in alveolar macrophages.

Figure 5. VIP inhibited macrophage polarization towards M1 and promoted polarization towards M2 induced by LPS or IL-4-induced. (A-G) Immunofluorescence shows the expression levels of the M1 macrophage markers CD86 and IL-6, as well as the M2 macrophage markers CD206 and IL-10. (G-H) Real-time PCR analysis shows CD86 and CD206 mRNA expression levels. All data are expressed as mean \pm S.D. $(n=3)$. \#\# $p<0.01$,\#\#\# $p<0.001$ vs. control group. ${ }^{*} p<0.05, * \star p<0.01$ vs. LPS group. $+p<$ $0.05,++p<0.01,+++p<0.001$ vs. IL-4 group.

\section{Pseudoephedrine + emodin treatment induces VIP/cAMP/PKA signaling pathway and inhibits NF-KB signaling pathway}

Previous results have shown that VIP can inhibit M1 polarization and promote M2 polarization in alveolar macrophages, thereby showing anti-inflammatory results. In the present study, we examined the expression of the VIP/cAMP/PKA signaling pathway and the NF-KB signaling pathway in the lung tissues of LPS-induced rats after treatment with pseudoephedrine + emodin.

Results are shown in Fig. 6. Pseudoephedrine + emodin treatment effectively promoted VIP protein and mRNA expressions both in serum and in lung tissues (Fig. 6A-D), elevated cAMP and p-PKA protein expression in lung tissues (Fig. 6C-E), and significantly inhibited NF-KB phosphorylation.

This result indicates that pseudoephedrine + emodin treatment exerts anti-inflammatory effects via the VIP/cAMP/PKA signaling pathway.

Figure 6. Pseudoephedrine + emodin increased VIP/cAMP/PKA pathways and inhibited NF-KB in LPSinduced ALI rats. (A) ELISA results for VIP in serum. (B) Real-time PCR analysis results for lung tissue 
mRNA expression. (C) Western blot results for protein expression. (D) Western blot results for VIP and CAMP protein expression. (E-F) Western blot results for the phosphorylation of PKA, IKBa and P65. All data are expressed as mean \pm S.D. $(n=3)$.\#\# $p<0.01$, \#\#\# $p<0.001$ vs. control group. ${ }^{\star} p<0.05$, ${ }^{* \star} p<$ $0.01, * \star * p<0.001$ vs. LPS group.

\section{Pseudoephedrine + emodin treatment inhibits M1 polarization and promotes $\mathrm{M} 2$ polarization in alveolar macrophages}

$\mathrm{F} 4 / 80$ is a macrophage surface marker that is commonly used to reflect macrophage activation status and recruitment levels. To determine the effects of pseudoephedrine + emodin on the polarization of macrophages in LPS-induced rat lung tissues, we performed immunohistochemical analysis of $F 4 / 80$ on rat lung tissue sections (Fig. 7A-B) and performed immunofluorescence analysis of CD80, IL-13; F4/80, IL10 (Fig. 7C-H).

Results indicate that the macrophage surface marker F4/80 was highly expressed in both the LPS group and the treatment group compared with the control group. However, macrophages in the LPS group were mainly M1-polarized and formed an inflammatory environment in the lung tissue, whereas the treatment group showed inhibited M1 polarization and increased M2 polarization, as well as lower expression of CD80 and IL-1 $\beta$, and higher expression of IL-10, which, taken together, formed an anti-inflammatory environment.

When taken together with all aforementioned results, this indicates that pseudoephedrine + emodin treatment can inhibit M1 polarization and promoted M2 polarization via the VIP/CAMP/PKA signaling pathway, thereby producing anti-inflammatory effects.

Figure 7. Pseudoephedrine + emodin inhibited M1 macrophage and activated M2 macrophage in lung tissues.

(A) Immunohistochemistry shows F4/80 expression levels. (B-C) Immunofluorescence shows CD80, IL-1B, $\mathrm{IL}-10$, and F4/80 expression levels. All data are expressed as mean \pm S.D. $(n=3)$.\#\# $p<0.01$, \#\#\#p< 0.001 vs. control group. ${ }^{\star * \star} p<0.001$ vs. LPS group.

\section{Discussion}

Inflammation is an important component of the immune response that protects the body from pathogens but inflammatory overreaction could lead to tissue and organ damage (Serhan et al., 2008). Increased and prolonged inflammation in ALI pathogenesis can lead to lung tissue damage (Kylanpaa et al., 2010). ALI could also lead to blood barrier dysfunction and increased capillary basement membrane permeability in the lungs, with protein fluid leaking into the interstitial space and causing pulmonary oedema (Li et al., 2018). NLRP3 inflammasomes activate the pro-inflammatory IL-1 $\beta$ in a caspase-1- 
dependent manner, which exacerbates inflammatory response and induces inflammatory cell death (Chen et al., 2018).

In our study, we treated rats with pseudoephedrine + emodin to effectively reduce the level of NLRP3 and the levels of the inflammatory TNF- $\alpha$ and IL-1 $\beta$, thereby achieving effective control of inflammatory overreaction and lung injury. Fever, a classic clinical presentation of inflammation, was found in ALI rats between two to eight hours after injection with LPS and treatment with pseudoephedrine + emodin was effective in relieving febrility.

We also demonstrated that pseudoephedrine + emodin could increase the expression of AQP-1 and AQP-5 proteins in the lung tissue homogenate of LPS-induced ALI rats. The dynamic equilibrium of water is the basis of cell survival and the transport of water across the cell membrane is controlled by AQP. AQP-1 and AQP-5 have similar structural features. AQP-1 is mostly distributed in the capillaries, lymphatic vessels and mesothelial cells of the respiratory tract and mainly regulate interstitial lung oedema that result from increased pressure in the pulmonary circulation. In contrast, AQP-5 is highly expressed in saliva, sweat, lacrimal glands, and in the lungs and airways. In lung tissues, AQP-5 is mainly found in type I alveolar epithelial cells and airway secretory gland cells, is closely related to the transmembrane transport of water molecules in epithelial cells, and plays a key role in the secretion of isotonic fluid (Raina., 1995). We found that D/W ratio was higher in rats treated with pseudoephedrine + emodin than in the LPS group, with indicates that the treatment was effective in alleviating inflammatory exudative pulmonary oedema in lung tissues (Fig. $1 \mathrm{H}-\mathrm{J})$.

Polarized M2 macrophages play a critical role in suppressing excessive inflammatory responses (Quinton et al., 2018). VIP, as an anti-inflammatory neuropeptide, plays an important role in immune regulation (Zhou et al., 2019). VIP acts as a negative regulator of inflammation by regulating the functioning and distribution of monocyte, macrophage, and regulatory $\mathrm{T}$ cells, thereby generating an anti-inflammatory microenvironment (Ramhorst et al., 2019). Through cellular experiments, we demonstrated that VIP effectively inhibited the expression of the pro-inflammatory TNF-a and IL-6 in M1 macrophages, and the expression of iNOS mRNAs induced by TNF-a, while simultaneously increasing the expression of the antiinflammatory IL-10, Arg-1, and Ym-1 proteins and mRNAs in M2 macrophages. Subsequent immunofluorescence results reinforced the regulatory role of VIP in inhibiting M1 polarization and promoting M2 polarization in macrophages (Fig. $5 \mathrm{C}$ ). Various inflammatory mediators on the NF-KB pathway play a crucial role in the pathogenesis of ALI, and the NF-KB pathway is key to the activation of NLRP3 and pro-inflammatory factors (Tate et al., 2018; Grebe et al., 2018; Kang et al., 2001). Studies have shown that VIP can elevate cAMP and p-PKA expression levels by binding to the VPAC1 receptor of M1 macrophages polarized in vitro, which in turn downregulates the transcriptional activity of the NF-KB (p65) signaling pathway by inhibiting IkBa phosphorylation (Mar et al., 2016). In our study, we confirmed through Western blot that treatment with pseudoephedrine + emodin activates LPS-induced VIP/cAMP/PKA signaling pathways in rat lung tissues, thereby blocking gene transcription in the NF-KB (p65) signaling pathway (Fig. 6A-F). Immunohistochemical and immunofluorescence results of lung tissues showed that both the LPS group and the treatment group exhibited elevated expression of the 
macrophage marker F4/80 compared with the control group; however, M1 macrophage activation was predominant in the LPS group, whereas M2-type macrophage activation was predominant in the treatment group, with the inflammatory environment within lung tissues being significantly suppressed in the treatment group (Fig. 7).

Figure 8. An anti-inflammatory mechanism model of NF-KB activation inhibited by activating the VIP/ cAMP /PKA pathway in ALI rats.

\section{Conclusions}

Our study proved that the combination of pseudoephedrine hydrochloride and emodin was effective in ameliorating LPS-induced ALI in rats by inducing VIP/CAMP/PKA signaling, inhibiting the NF-KB inflammatory pathway, suppressing macrophage M1 polarization, and promoting macrophage M2 polarization. Inthe further studies, we intend to consider additional perspectives for ameliorating ALI, such as attempting to regulate macrophage polarization through glycogen metabolism and its mediated inflammatory responses (Ma et al., 2020).

\section{Abbreviations}

ALI

Acute lung injury; LPS:Lipopolysaccharide; IL-6:Interleukin-6; TNF-a:Tumor necrosis factor-a; GCSF:Granulocyte colony-stimulating factors ; VIP:Vasoactive intestinal peptide; cAMP:Cyclic adenosine monophosphate; ACs:Activate adenylate cyclases; PKA:Protein kinase; ARIs:Acute respiratory infections; BALF:Bronchoalveolar lavage fluid; H\&E:Haematoxylin and eosin; ELISA:Enzyme-linked immunosorbent assay; WB:Western blotting;

\section{Declarations}

\section{Acknowledgements}

We thank LetPub (http://www.LetPub.com) for linguistic assistance during the preparation of this manuscript.

\section{Authors' contributions}

SY and WW designed the study; WW wrote the main manuscript text; WW, JS, YH, and JL performed the animal experiments; WW, SY, and XW analysed the data and prepared the fgures. All authors read and approved the final manuscript.

\section{Funding}

This study was supported by the National Natural Science Foundation of China (81703974) and Discipline innovation team construction project of Shaanxi University of Chinese medicine (2019-YL05) 
Availability of data and materials

The datasets used in the current study are available from the corresponding author on reasonable request.

\section{Ethics approval and consent to participate}

The animal care and experimental procedures used in the current study were approved by the Institutional Animal Care and Use Committee of the Shaanxi University of Chinese Medicine.

\section{Competing interests}

The authors declare that they have no competing interests.

\section{Author details}

${ }^{1}$ College of Basic Medicine, The Shaanxi University of Chinese Medicine, Xianyang 712046, China;

E-Mails: Ysg2002.student@sina.com(S.-G.Y.);949222779@qq.com(W.-B.W.); drhy1982@126.com(Y.H.)

${ }^{2}$ Departments of infectious disease, The Affliated Hospital of Shaanxi University of Chinese Medicine,Xianyang,China; E-mails:Lijingtao555@163.com(J.-T.L.)

${ }^{3}$ Departments of respiratory diseases The Affliated Hospital of Shaanxi University of Chinese Medicine,Xianyang,China; E-mails $₫ 2780067265 @ q q . c o m(J . S$.

${ }^{4}$ Department of Endocrinology, Genetics and Metabolism, Xi 'an Children's Hospital,Xi'an,China;Emails:4067010@qq.com(X.-Y.W.)

\section{References}

1. Chen J, Chen ZJ. Ptdlns4P on dispersed trans-Golgi network mediates NLRP3 inflammasome activation. Nature. 2018;564:71-6.

2. Dong X, Fu J, Yin X, Cao S, Li X, Lin L, Huyiligeqi, Ni J. Emodin: A Review of its Pharmacology, Toxicity and Pharmacokinetics. Phytother Res. 2016;30:1207-18.

3. DERWALL M, MARTIN L, ROSSAINT R. The acute respiratory distress syndrome: Pathophysiology, current clinical practice, and emerging therapies. Expert Rev Respir Med. 2018;12:1021-9.

4. Gupta N, Su X, Popov B, et al. 2007.Intrapulmonary delivery of bone marrow-derived mesenchymal stem cells improves survival and attenuates endotoxin induced acute lung injury in mice. $\mathrm{J}$ Immunol.179: 1855-1863.

5. Grebe A, Hoss F, Latz E. NLRP3 Inflammasome and the IL-1 Pathway in Atherosclerosis. Circ Res. 2018;122:1722-40. 
6. Huang $X$, Zeng $Y$, Jiang $Y$, et al. Lipopolysaccharide- binding protein downregulates fractalkine through activation of p38 MAPK and NF-Kb. Mediators Inflamm. 2017;2017:9734837.

7. Juana M, Gancedo. Biological roles of cAMP: variations on a theme in the different kingdoms of life. Biological Reviews. 2013;88:645-68.

8. Karpurapu M, Lee YG, Qian Z, Wen J, et al. Inhibition of nuclear factor of activated T cells (NFAT) c3 activation attenuates acute lung injury and pulmonary edema in murine models of sepsis. Oncotarget. 2018;9:10606-20.

9. Kylanpaa ML, Repo H, Puolakkainen PA. Inflammation and immunosuppression in severe acute pancreatitis. World J Gastroenterol. 2010;16:2867-72.

10. Kang JL, Lee HW, Lee HS, Pack IS, Chong Y, Castranova V, Koh Y. Genistein prevents nuclear factorkappa B activation and acute lung injury induced by lipopolysaccharide. Am J Respir Crit Care Med. 2001;164:2206-12.

11. Laube BL. Aerosolized Medications for Gene and Peptide Therapy. Respir Care. 2015;60:806-21. discussion 821-4.

12. Liu W, Liu K, Zhang S, et al. Tetramethylpyrazine Showed Therapeutic Effects on Sepsis-Induced Acute Lung Injury in Rats by Inhibiting Endoplasmic Reticulum Stress Protein Kinase RNA-Like Endoplasmic Reticulum Kinase (PERK) Signaling-Induced Apoptosis of Pulmonary Microvascular Endothelial Cells. Med Sci Monit. 2018;24:1225-31.

13. Lin YC, Chang CW, Wu CR. Antitussive, anti-pyretic and toxicological evaluation of Ma-Xing-Gan-ShiTang in rodents. BMC Complement Altern Med Nov. 2016;16:456.

14. Li P, Zhang J, Liang X. Characterization of the complete chloroplast genome of Ephedra sinica Stapf (Ephedraceae), a traditional Chinese medicine. Mitochondrial DNA B Resour. 2019;4:3301-2.

15. LaForce C, Gentile DA, Skoner DP. A randomized, double-blind, parallel-group, multicenter, placebocontrolled study of the safety and efficacy of extended-release guaifenesin/pseudoephedrine hydrochloride for symptom relief as an adjunctive therapy to antibiotic treatment of acute respiratory infections. Postgrad Med. 2008;120:53-9.

16. Lu Y, Jiang Y, Ling L, Zhang Y, Li H, Chen D. Beneficial effects of Houttuynia cordata polysaccharides on "two-hit" acute lung injury and endotoxic fever in rats associated with anti-complementary activities. Acta Pharm Sin B. 2018;8:218-27.

17. Li Y, Li H, Liu S, et al. Pirfenidone ameliorates lipopolysaccharide-induced pulmonary inflammation and fibrosis by blocking NLRP3 inflammasome activation. Mol Immunol. 2018;99:134-144.

18. Locati M, Curtale G, Mantovani A. Diversity, Mechanisms, and Significance of Macrophage Plasticity. Annual Review of Pathology Mechanisms of Disease. 2019;15:1-25.

19. MATTHAY M A, ZEMANS R L, ZIMMERMAN GA, et al. Acute respiratory distress syndrome. Nat Rev Dis Primers. 2019;5:18.

20. Ma J, Wei K, Liu J, Tang K, Zhang H, Zhu L, Chen J, Li F, et al. Glycogen metabolism regulates macrophage-mediated acute inflammatory responses. Nat Commun. 2020;11:1769. 
21. Ma LQ, Pan CS, Yang N, Liu YY, et al. Posttreatment with Ma-Xing-Shi-Gan-Tang, a Chinese medicine formula, ameliorates lipopolysaccharide-induced lung microvessel hyperpermeability and inflammatory reaction in rat. Microcirculation. 2014;21:649-63.

22. Mar Carrión S, Pérez-García C, Martínez, et al. VIP impairs acquisition of the macrophage proinflammatory polarization profile. J Leukoc Biol. 2016;100:1385-93.

23. Mellett M, Atzei P, Jackson R, et al. Mal mediates TLR-induced activation of CREB and expression of IL-10. J Immunol. 2011;186:4925-35.

24. Murray PJ. Macrophage Polarization Annual Review of Physiology. 2016;79:541.

25. Onoue S, Matsui T, Kato M, Mizumoto T, et al. Chemical synthesis and formulation design of a PEGylated vasoactive intestinal peptide derivative with improved metabolic stability. Eur J Pharm Sci. 2013;49:382-9.

26. Quinton LJ, Walkey AJ, Mizgerd JP. Integrative Physiology of Pneumonia. Physiological Reviews. 2018;98:1417-64.

27. Pearce L, Komander D, Alessi. Alessi D DR. The nuts and bolts of AGC protein kinases. Nat Rev Mol Cell Biol. 2010;11:9-22.

28. Raina S. Molecular cloning and characterization of an aquaporin cDNA from salivary, lacrimal, and respiratory tissues. J Biol Chem. 1995;270:1908-12.

29. Ramhorst R, Calo G, Paparini $D$, et al. Control of the inflammatory response during pregnancy: potential role of VIP as a regulatory peptide. Ann N Y Acad Sci. 2019;1437:15-21.

30. Szapiel SV, Elson NA, Fulmer JD, Hunninghake GW, Crystal RG. Bleomycin-induced interstitial pulmonary disease in the nude, athymic mouse. Am Rev Respir Dis. 1979;120:893-9.

31. Shi DF, Zheng MZ, Wang YM, et al. Protective effects and mechanisms of mogroside $V$ on LPSinduced acute lung injury in mice. Pharm Biol. 2014;52:729-34.

32. Soni M, Carabin I, Griffiths JC, Burdock G. Safety of ephedra: Lessons learned. Toxicol Lett. 2004;150:97-110.

33. Serhan CN, Chiang N, Van Dyke TE. Resolving inflammation: dual anti-inflammatory and proresolution lipid mediators. Nat Rev Immunol. 2008;8:349-361.

34. Tate MD, Mansell A. An update on the NLRP3 inflammasome and influenza: the road to redemption or perdition? Curr Opin Immunol. 2018;54:80-5.

35. Ware LB, Matthay MA. The acute respiratory distress syndrome. N Engl J Med. 2000;342:1334-49.

36. Wiersinga WJ, Rhodes A, Cheng AC, et al. Pathophysiology, Transmission, Diagnosis, and Treatment of Coronavirus Disease 2019 (COVID-19): A Review. JAMA The Journal of the American Medical Association. 2019;324:782-93.

37. Xu C, Wang Y, Sun R, Qiao X, et al. Modulatory effffects of vasoactive intestinal peptide on intestinal mucosal immunity and microbial community of weaned piglets challenged by an enterotoxigenic Escherichia coli (K88). PLoS One. 2014;9:e104183. 
38. Zhang B, Liu ZY, Li YY, et al. Antiinflammatory effects of matrine in LPS-induced acute lung injury in mice. Eur J Pharm Sci. 2011;44:573-579.

39. Zhou Y, Zhang CY, Duan JX, et al. Vasoactive intestinal peptide suppresses the NLRP3 inflammasome activation in lipopolysaccharide-induced acute lung injury mice and macrophages. Biomed Pharmacother. 2019;121:109596. 